\title{
SUNSPOT MOTIONS FROM A STUDY OF KODAIKANAL AND MOUNT WILSON OBSERVATIONS
}

\author{
ROBERT F. HOWARD \\ National Solar Observatory \\ National Optical Astronomy Observatories $\dagger$ \\ Tucson, Arizona 85726, USA \\ K. R. SIVARAMAN \\ S. S. GUPTA \\ Indian Institute of Astrophysics \\ Bangalore 560 034, India \\ PAMELA I. GILMAN \\ Mount Wilson Observatory \\ And Department of Astronomy \\ University of California, Los Angeles \\ Los Angeles, California 90024, USA
}

\begin{abstract}
A study of the daily motions of individual sunspots and of sunspot groups has been undertaken using Kodaikanal and Mount Wilson white-light observations. The Mount Wilson measurements were completed several years ago, and the Kodaikanal measurements have recently been started, using a technique identical to that used for the Mount Wilson data. Both data sets started early in this century, and both have continued to the present time. Preliminary results of a comparison of the two data sets are presented which show a good agreement between them in spot areas and motions. Analysis of the combined data set for the years that are available so far indicates that many more spots are available in such a reduction because the 12-hour time difference between the two sites allows for a more certain identification of individual spots from one observation to the next than in the case when data from only one site are used. This greatly increases the number of sunspots available for motion studies, particularly the smaller sunspots. Preliminary rotation and latitude drift reduction from the combined data set confirm earlier results from the Mount Wilson data alone.
\end{abstract}

\section{Introduction}

The important processes that produce the solar activity cycle take place beneath the solar surface. One method to advance our knowledge of the cycle mechanism is to study the motions at the surface of tracers that are rooted below. Considerable effort has gone into this activity in recent years (e.g. Schröter, 1985).

† Operated by the Association of Universities for Research in Astronomy, Inc., under Contract with the National Science Foundation.

E. R. Priest and V. Krishan (eds.), Basic Plasma Processes on the Sun, 107-111. (C) 1990 IAU. Printed in the Netherlands. 
Several years ago the Mount Wilson white-light photoheliogram data set was measured for positions and areas of individual sunspots. This program resulted in a number of studies of properties of spots and spot groups (Howard, Gilman, and Gilman, 1984; Gilman and Howard, 1984a; 1984b; Gilman and Howard, 1985; 1986: Howard and Gilman, 1986; Bogdan, et al., 1988). A similar data set has been accumulated at the Kodaikanal site of the Indian Institute of Astrophysics. This set of plates extends back to 1905 and is in excellent condition.

Recently a digitizing instrument similar to that used for the Mount Wilson data was installed at Kodaikanal, and measurements of sunspots were begun. It is planned to measure every one of the plates at Kodaikanal from 1905 to the present. The technique being used is as nearly identical as possible to that used for the Mount Wilson plates and described in the first of the series of papers that came from this work (Howard, Gilman, and Gilman, 1984).

The measurement of the Kodaikanal white-light plates will soon give us a valuable data set with which to study the motions of sunspots at the solar surface and the radius of the sun during this century. This will be the largest collection of individual sunspot position measures in existence. The Greenwich data set, which has been much used in studies of sunspot motions, is an invaluable set of excellent data covering spot group areas and positions, but it does not have a complete set of individual spot area results. It has, however, been used to study the motions of single-spot groups (e.g. Wöhl, 1988).

The work is now in progress and so far several years of data have been measured. In this paper we discuss the measurements and present some of the first results from the Kodaikanal measures and from the combined Kodaikanal-Mount Wilson data set.

\section{Observations, Measurements, and Reduction}

Daily white-light photographs of the full-disk sun have been taken at Kodaikanal since 1905. These observations have been made with the same instrument and using the same observing procedures during the entire interval. The image size is approximately $20 \mathrm{~cm}$, which is slightly larger than the Mount Wilson images.

The measuring technique for the Kodaikanal plates is quite similar to that used for the Mount Wilson plates. This is done in order to provide a uniform data set for comparison of the Kodaikanal results with the Mount Wilson results and for ease of combining the two data sets. A digitizing pad which is operated by a cursor containing a crosshair is used, as has been described for the Mount Wilson measurements (Howard, Gilman and Gilman, 1984). The accuracy of the digitizer is several times better than that of the best seeing on any of the solar images, and is therefore not a factor in the reduction process.

The measurement of a plate for a day consists of making 8 evenly spaced determinations of the limb position and then two position measures for each sunspot on the disk within $60 \mathrm{deg}$ of the central meridian.

Each year is reduced separately as a whole. Accurate corrections to the positions of the limb points and the spot measures are made for atmospheric refraction using the time and date of the observation. The radius and central position of the solar disk are derived in digitizing board coordinates. Then solar coordinates are derived for each spot measurement point. Areas and positions of the spots are derived. Areas are corrected for projection effects. 
With the data for each day tabulated, a search for consecutive day's observations is made. In the case of the combined data set from both observatories, the next available observation is used. The majority of the time differences in the combined data set cluster around 1/2 day. In practice, an upper limit of 1.5 days is used for the determination of 'daily' motions.

For each consecutive pair of observations, differences in latitude and CMD are calculated for each spot group and for each spot. In the case of groups the positions are area-weighted. The technique for determining the identification of individual sunspots is described (Howard, Gilman, and Gilman, 1984).

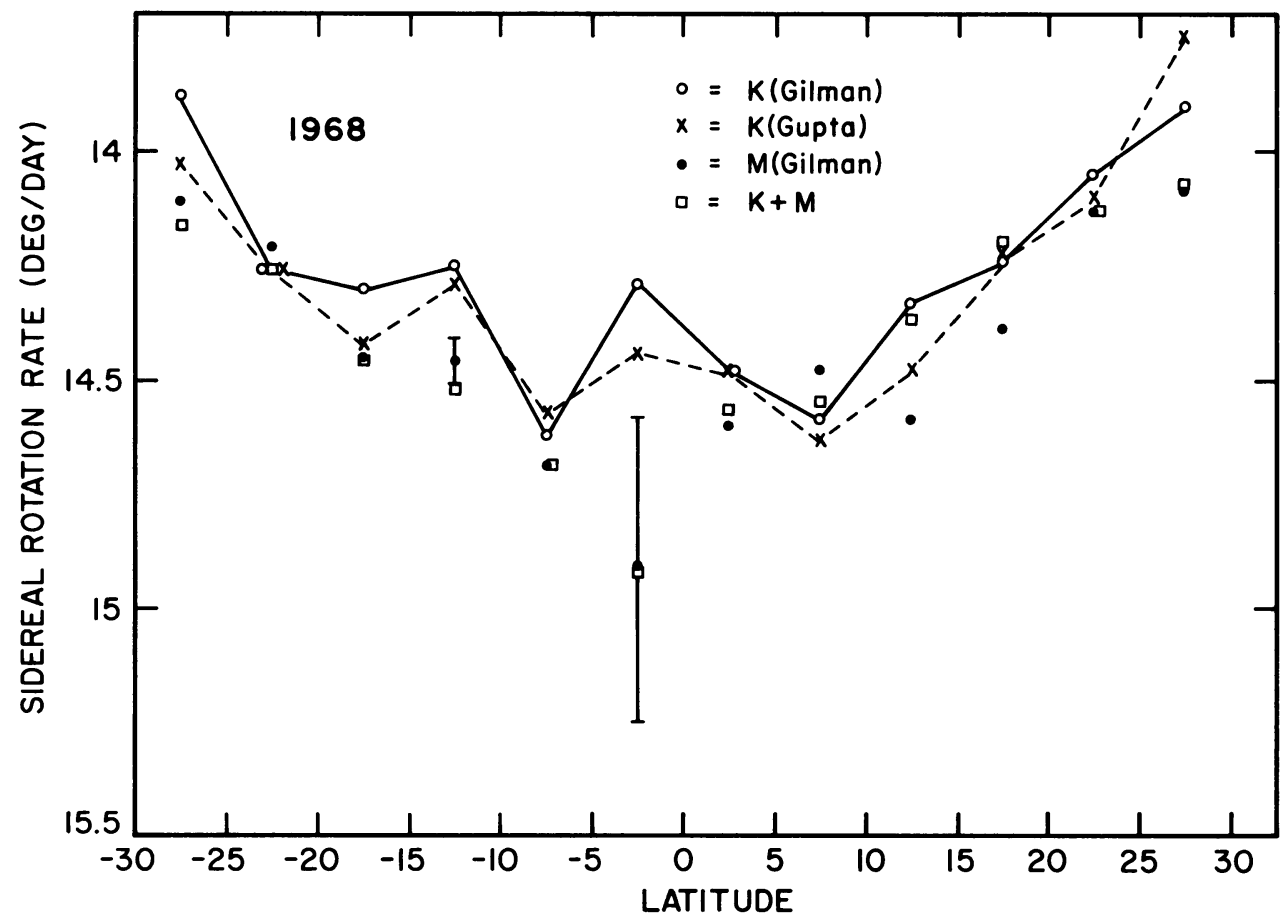

Figure 1. Sidereal rotation rate as a function of latitude for sunspots for the first 9 months of 1968. Circles are Kodaikanal data measured by Gilman; crosses are Kodaikanal data measured by Gupta; solid circles are Mount Wilson data; and squares are combined Kodaikanal-Mount Wilson data (Kodaikanal data measured by Gupta). Typical error bars are shown for low and intermediate latitudes. The errors at high latitudes are similar in magnitude to those at low latitudes.

\section{Results}

Figure 1 shows a comparison of solar rotation results for various data and various measurements for the first 9 months of 1968. Only part of the year was used because only that part of the year was available for the measurements of Kodaikanal data by PG. The 
Kodaikanal data are represented by open circles and crosses. The agreement between the measurements made by the two measurers is seen to be well within the limits of the errors. Error bars ( \pm one standard deviation) are shown for the Mount Wilson measures for low latitudes at 0 to $-5 \mathrm{deg}$ (typical also for high latitudes) and for intermediate latitudes at -10 to $-15 \mathrm{deg}$, where there are more spots. The low latitude error bars refer to an average over 11 sunspots, and the intermediate latitude error bars refer to an average over 264 sunspots. These errors are of similar magnitude for Kodaikanal measures and slightly lower for the combined data set.

The differential rotation curve in Figure 1 representing the combined data set is indicated by squares. For the combined data set we used the Kodaikanal measures of SSG. The combined results for any latitude do not always fall between the results from the two separate measurements because it is not a simple average of the two sets. With many 1/2-day determinations in the combined result, this means that there are many individual determinations in this result that are not in the individual observatory results.

We determined the value of $A$, the sidereal equatorial rotation velocity in the representation $\omega=A+B \sin ^{2} \phi$, where $\phi$ is the solar latitude. For the full disk data averaged over the 9-month interval, this value is $14.39 \mathrm{deg} / \mathrm{day}$ for the Mount Wilson data, 14.52 for the Kodaikanal data measured by PG, 14.62 for the Kodaikanal data measured by SSG, and 14.64 for the combined data set (Kodaikanal data measured by SSG). For the full 1921 through 1982 interval of the Mount Wilson data published earlier (Howard, Gilman, and Gilman, 1984) $A$ is 14.522 , although there were significant variations from year to year.

The results of the Kodaikanal measurements so far are encouraging, and we anticipate that we will have an opportunity soon to add significantly to our knowledge of solar dynamics.

\section{Acknowlegements}

This research has been made possible through a Grant from the US-India Cooperative Science Program of the Division of International Programs, US National Science Foundation. We gratefully acknowledge assistance from a number of people, including A. V. Ananth and R. Srinivasan, of the Indian Institute of Astrophysics, John E. Boyden of the University of California, Los Angeles, and Stephen A. Colley of the National Solar Observatory.

\section{References}

Bogdan, T. J., Gilman, P. A., Lerche, I., and Howard, R.: 1988, Astrophys. J. 327, 451.

Gilman, P. A., and Howard, R.: 1984a, Astrophys. J. 283, 385.

Gilman, P. A., and Howard, R.: 1984b, Solar Phys. 93, 171.

Gilman, P. A., and Howard, R.: 1985, Astrophys. J. 295, 233.

Gilman, P. A., and Howard, R.: 1986, Astrophys. J. 303, 480.

Howard, R., Gilman, P. A., and Gilman, P. I.: 1984, Astrophys. J. 283, 373.

Howard, R., and Gilman, P. A.: 1986, Astrophys. J. 307, 389.

Schröter, E. H.: 1985, Solar Phys. 100, 141.

Wöhl, H.: 1988, Solar Phys. 116, 199. 


\section{DISCUSSION}

PRIEST: (i) What do you believe is the cause for small spots to rotate faster than large ones? Is it because the small ones are affected more by the photospheric plasma?

(ii) Are there implications for the validity or otherwise of Piddington's tree-trunk model of the subphotospheric structure?

HOWARD: (i) I believe that the important factor is probably the age of the spot. This is not really an answer to this question, but one can imagine a weakening of the connection between the lower and surface layers as the spot ages and grows. Thus the spot would tend to rotate closer to the surface plasma rate as it aged.

(ii) It was this model that led me to assume that each spot group rotated as a whole i.e. showed no differential rotation. The fact that this is not the case implies either that the tree trunk model is not valid or that the connection of the spots to the subsurface layer is weak. 